\title{
PERIOD-PRESERVING VARIATION OF A RIEMANN SURFACE
}

\author{
Dedicated to Professor Mitsuru Nakai on his sixtieth birthday
}

\author{
By MaSAhiKo TANiguCHI
}

\section{Introduction.}

In the Teichmüller space of a given surface, the sub-locus, consisting of all marked Riemann surfaces which admit holomorphic abelian differentials having the prescribed periods, plays several important roles in the theory of Riemann surfaces.

In this note, we will introduce explicit local parameters at a generic point of this sub-locus. They indicate how zeros of differentials with prescribed periods vary on the surfaces.

\section{$\S 1$. The fundamental surgery.}

Let $\alpha=r e^{i \theta} \in C(r>0,0 \leqq \theta \leqq 2 \pi)$ be arbitrarily given. Then a surgery of $C$ which preserves the differential $z d z$ can be defined as follows: First, set $D(\alpha)=C-\left\{z=t \cdot r^{1 / 2} e^{i \theta / 2} \mid-1 \leqq t \leqq 1\right\}$. Then $D(\alpha)$ is mapped conformally onto $D(-\alpha)=C-\left\{w=i t \cdot r^{1 / 2} e^{i \theta / 2} \mid-1 \leqq t \leqq 1\right\}$ by the mapping

$$
w=\frac{1}{2}\left(\zeta-\frac{\alpha}{\zeta}\right) \quad \text { with } \quad z=\frac{1}{2}\left(\zeta+\frac{\alpha}{\zeta}\right),
$$

namely,

$$
w=F(z)=z \cdot\left(1-\frac{\alpha}{z^{2}}\right)^{1 / 2},
$$

where we take the branch of $\left(1-\alpha / z^{2}\right)^{1 / 2}$ such that $1^{1 / 2}=1$.

Then $w=F(z)$ maps $D(\alpha)$ onto $D(-\alpha)$. Furthermore, a simple computation shows that

$$
w d w=z d z \text {. }
$$

Definition. We call this surgery the fundamental surgery (of the differential $z d z$ ) at $z=0$ with respect to $\alpha$.

Remark. This is a kind of Schiffer's interior variation, and can be regarded also as the branch-point variation with respect to the branched covering projec-

Received June 16, 1993. 
tion $\pi(z)=z^{2}$ (where the branch point $z=0$ moves to $\alpha$, and hence $w^{2}=z^{2}-\alpha$ ).

Recall that such variation can be represented as a quasiconformal deformation (cf. [2]). For instance, assuming that $r<\rho$, we expand $F$ as

$$
F(z)=z+\sum_{n=0}^{\infty} c_{n} z^{-n}
$$

on $\{\rho<|z|<+\infty\}$. Then the mapping

$$
f(z)=\left\{\begin{array}{lll}
z+\sum_{n=0}^{\infty} c_{n}\left(\bar{z} / \rho^{2}\right)^{n} & \text { on } & \{|z| \leqq \rho\} \\
F(z) & \text { on } & \{|z|>\rho\}
\end{array}\right.
$$

is a quasiconformal selfmapping of $C$, and the complex dilatation $\mu_{f}(z)$ of $f$ is

$$
\left\{\begin{array}{lll}
\sum_{n=1}^{\infty} n c_{n} \rho^{-2 n} \bar{z}^{n-1} & \text { on } & \{|z| \leqq \rho\} \\
0 & \text { on } & \{|z|>\rho\} .
\end{array}\right.
$$

Since $c_{1}=-\alpha / 2$ and $\mu_{f}=c_{1} \rho^{-2} \cdot \chi_{\rho}+O\left(|\alpha|^{2}\right.$ ) (which holds uniformly with respect to $z$ ) as $\alpha$ tends to 0 , we have

$$
\mu_{\alpha}(z) \quad\left(=\left.\frac{\partial \mu_{f}}{\partial \alpha}\right|_{a=0}(z)\right)=-\frac{\rho^{-2}}{2} \chi_{\rho}(z)
$$

where $\chi_{\rho}(z)$ is the characteristic function of the disc $\{|z| \leqq \rho\}$.

\section{$\S 2$. Period-preserving variation.}

Fix a compact Riemann surface $R$ of genus $g \geqq 2$, and a holomorphic abelian differential $\varphi$ on $R$. Also we denote by $T(R)$ the Teichmüller space of $R$ (, see for instance, [4]).

Suppose that $\varphi$ has only simple zeros $\left\{p_{j}\right\}_{j=1}^{2 g-2}$. For every $p_{\jmath}$, fix a suitable local parameter $z_{j}$ on a neighborhood $U_{j}$ of $p_{j}$ such that $z_{j}\left(U_{\jmath}\right)=\left\{\left|z_{\jmath}\right|<2 \rho_{j}\right\}$ and $\varphi$ can be represented as $z_{j} d z_{\text {, on }}\left\{\left|z_{j}\right|<2 \rho_{j}\right\}$. We also assume that $\left\{U_{j}\right\}_{j=1}^{q^{g}-2}$ are mutually disjoint.

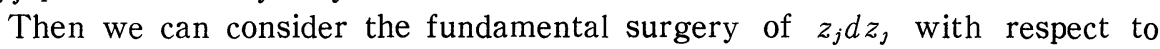
$\alpha_{j} \in C$ with $\left|\alpha_{j}\right|<\rho_{j} / 2$. Let $w_{j}=F_{j}\left(z_{j}\right)$ be the corresponding conformal map as in $\S 1$. Then by deleting all topological disc $\left\{p \in R|| z_{j}(p) \mid<\rho_{j}\right\}$ from $R$ and by pasting different topological $\operatorname{discs} F_{j}\left(\left\{\left|z_{j}\right|<\rho_{j}\right\}\right)$ in the $w_{j}$-plane naturally, we get a marked Riemann surface $R_{\alpha}$, or equivalently a point $\left[R_{\alpha}\right]$ of $T(R)$, where we set $\alpha=\left(\alpha_{1}, \cdots, \alpha_{2 g-2}\right)$. It is clear that the complex structure of $R_{\alpha}$ may differs from that of $R$ in general. Nevertheless, $\varphi$ determines uniquely a holomorphic abelian differential $\varphi_{\alpha}$ on $R_{\alpha}$, which has the same periods as $\varphi$.

Definition. We call the above surgery of $R$ for given $\varphi$ and $\alpha$ (with a sufficiently small $|\alpha|=\left\{\sum_{j=1}^{2 g-2}\left|\alpha_{j}\right|^{2}\right\}^{1 / 2}$ ) period-preserving variation associated with $\varphi$ with respect to the parameter $\alpha$. 
Remark. Period-preserving variation can be considered even for any holomorphic abelian differential on an arbitrary (not necessarily finite) Riemann surface.

Define a marking-preserving quasiconformal mapping $f[\alpha]: R \rightarrow R_{\alpha}$ similarly as in $\S 1$ on each topological disc $\left\{p \in R|| z_{j}(p) \mid<2 \rho_{\jmath}\right\}$. Let $\mu(\alpha)$ be the Beltrami differential of $f[\alpha]$. Then we have

$$
\begin{aligned}
\iint_{R} \mu_{\alpha_{\jmath}} \cdot \psi & =-\frac{\rho^{-2}}{2} \iint_{\left(\left|z_{\jmath}\right|<\rho_{j}\right\}} \psi_{j}\left(z_{\jmath}\right) d x_{j} d y_{\jmath} \quad\left(z_{\jmath}=x_{j}+\imath y_{\jmath}\right) \\
& =-\frac{\pi}{2} \phi\left(p_{\jmath}\right)
\end{aligned}
$$

for every holomorphic quadratic differential $\phi$ on $R$, where writing $\phi=\phi_{j}\left(z_{\jmath}\right) d z_{j}{ }^{2}$ on $\left\{\left|z_{j}\right|<\rho_{\jmath}\right\}$, we set $\left.\phi^{\prime} \phi_{j}\right)=\phi_{j}(0)$. In particular, we have

$$
\iint_{R} \mu_{\alpha} \cdot\left(\varphi \theta_{k}\right)=0 \quad(\jmath=1, \cdots, 2 g-2 ; k=1, \cdots, g)
$$

for any base $\left\{\theta_{k}\right\}_{k=1}^{g}$ of holomorphic abelian differentials on $R$.

Thus, though $\alpha$ moves in $C^{2 g-2}, R_{\alpha}$ moves at most in a (2g-3)-dimensional complex variety. We will show that, in case of holomorphic reproducing differential having only simple zeros on $R, R_{\alpha}$ can actually sweep a $(2 g-3)$ dimensional complex submanifold of $T(R)$.

To state the main theorem, fix a 1-cycle $c$ on $R$ and let $\sigma_{c}$ be the period reproducer for $c$, namely, $\left(\omega, \sigma_{c}\right)=\int_{c} \omega$ for every harmonic differential $\omega$ on $R$. Set $\theta_{c}=\theta_{c}[R]=\sigma_{c}+\imath^{*} \sigma_{c}$, and $W_{\theta_{c}}=\left\{\left[R^{\prime}\right] \in T(R) \mid R^{\prime}\right.$ admits a holomorphic abelian differential with the same periods as $\left.\theta_{c}\right\}$.

THEOREM 1. Suppose that $\theta_{c}$ has only simple zeros. Then, for every sufficiently small $\varepsilon>0$, the subset $W_{\varepsilon}=\left\{\left[R_{\alpha}\right] \in T(R)|| \alpha \mid<\varepsilon, \alpha_{2 g-2}=0\right\}$ is a $(2 g-3)$ dimensional complex submanifold of $W_{\theta_{c}}$, which gives a nerghborhood of the base point $[R]$ in $W_{\theta_{c}}$.

More precisely, $\left(\alpha_{1}, \cdots, \alpha_{2 g_{-3}}\right)$ gives a local parameter system of $W_{\theta_{c}}$ at $[R]$.

Remark. (1) Period-preserving variation is a special kind of deformation considered in [3], where the authors use, as models of the light-cone diagram, linear combinations of the Green's functions on $R$ in the sense of SchifferSpencer.

The assertion of Theorem 1 remains true for such functions with only simple zeros (cf. [6]).

(2) We can take $2 g$-periods of $\sigma_{c}$ as real local parameters transversal to $W_{\varepsilon}$ at $[R]$ in $T(R)$.

(3) The same assertion will be true for any holomorphic abelian differential with only simple zeros. 
To prove Theorem 1, we first recall the classical Accola's theorem [1], which states that critical horizontal trajectories of $\theta_{c}{ }^{2}$ cut $R$ into several doubly connected subdomains called characteristic ring domains. Also recall that this ring structure induced by $\theta_{c}^{2}$ varies continuously in both metrical and geometrical sense. For precise description, see for instance [5, §2 Proposition].

LEMMA 1. The map

$$
\Pi: \alpha \in E_{\varepsilon}=\left\{|\alpha|<\varepsilon, \alpha_{2 g-2}=0\right\} \longrightarrow\left[R_{\alpha}\right] \in W_{\theta_{c}}
$$

is injective for every sufficiently small $\varepsilon$.

Proof. If not, there is two distinct point $\alpha$ and $\alpha^{\prime}$ such that $\left[R_{\alpha}\right]=\left[R_{\alpha^{\prime}}\right]$. Then since $\left(\theta_{c}\right)_{\alpha}$ and $\left(\theta_{c}\right)_{\alpha^{\prime}}$ has the same periods and $R$ is compact, we can conclude that $\left(\theta_{c}\right)_{\alpha}=\left(\theta_{c}\right)_{\alpha^{\prime}}$. In particular, $\left(\theta_{c}\right)_{\alpha}^{2}$ and $\left(\theta_{c}\right)_{\alpha^{\prime}}^{2}$ induce the same ring structure. From the definition of period-preserving variation, this implies that $\alpha=\alpha^{\prime}$ when $|\alpha|\left(=\left|\alpha^{\prime}\right|\right)$ is sufficiently small. q. e. d.

Furthermore, we conclude that $\left(\theta_{c}\right)_{\alpha}=\theta_{c}\left[R_{\alpha}\right]$ for every $\alpha$. In fact, $\operatorname{Im}\left(\theta_{c}\right)_{\alpha}$ has the same periods as $\operatorname{Im} \theta_{c}[R]$, and it is well-known that the periods of $\operatorname{Im} \theta_{c}\left[R_{\alpha}\right]$ are independent of $\alpha$. Thus $\operatorname{Im}\left(\theta_{c}\right)_{\alpha}-\operatorname{Im} \theta_{c}\left[R_{\alpha}\right]$ is exact, and hence is identically 0 when $R$ is compact.

In the sequel of this section, fix a sufficiently small $\varepsilon$ so that Lemma 1 holds.

LEMMA 2. The image $W_{\varepsilon}=\Pi\left(E_{\varepsilon}\right)$ is a neighborhood of $[R]$ in $W_{\theta_{c}}$.

Proof. Fix a characteristic ring domain $A[R]$ of $\theta_{c}{ }^{2}$. Then because of metrical and geometrical continuity, there is a neighborhood $V$ of $[R]$ (in $T(R)$ ) such that $\theta_{c}\left[R^{\prime}\right]^{2}$ has a characteristic ring domain $A\left[R^{\prime}\right]$ corresponding to $A[R]$ and $\theta_{c}\left[R^{\prime}\right]$ has only simple zeros $\left\{p_{j}\left[R^{\prime}\right]\right\}_{j=1}^{2 g-2}$ for every $\left[R^{\prime}\right] \in V$, where we can choose the ordering of zeros so that each $p_{j}\left[R^{\prime}\right]$ varies continuously in a suitable sense for every $j$.

Now fix $\left[R^{\prime}\right]$ in $U=V \cap W_{\theta_{c}}$ arbitrarily. Taking a smaller $V$ if necessary, we may assume that some mutually corresponding zeros, say $p_{2 g-2}[R]$ and $p_{2 g-2}\left[R^{\prime}\right]$ stay on the boundaries of $A[R]$ and $A\left[R^{\prime}\right]$, respectively.

Identify $p_{2 g-2}[R]$ to $p_{2 g-2}\left[R^{\prime}\right]$, which is equivalent to set $\alpha_{2 g-2}=0$. Then we can further identify some part of $A[R]$ to some of $A\left[R^{\prime}\right]$ so that $\theta_{c}[R]$ coincides with $\theta_{c}\left[R^{\prime}\right]$.

This identification breaks at some zero of $\theta_{c}[R]$ or of $\theta_{c}\left[R^{\prime}\right]$. Here we may assume, without loss of generality, that this happens at a zero, say, $p_{1}\left[R^{\prime}\right]$ of $\theta_{c}\left[R^{\prime}\right]$. Take a path $l_{1}^{\prime}$ in $A\left[R^{\prime}\right] \cup\left\{p_{1}\left[R^{\prime}\right], p_{2 g-2}\left[R^{\prime}\right]\right\}$ connecting $p_{1}\left[R^{\prime}\right]$ to $p_{2 g-2}\left[R^{\prime}\right]$. Also let $l_{1}$ be a path on $R$ corresponding to $l_{1}^{\prime}$, which connects $p_{1}[R]$ to $p_{2 g-2}[R]$ suitably.

Set 


$$
\alpha_{1}=\int_{l_{1}} \theta_{c}[R]-\int_{l_{1}^{\prime}} \theta_{c}\left[R^{\prime}\right] .
$$

When $V$ is sufficiently small, $\left|\alpha_{1}\right|$ is small enough to apply the fundamental surgery (period-preserving variation) at $p_{1}[R]$ with respect to $\alpha_{1}$. Moreover from the construction, we can continue to extend the above identification until we identify $p_{1}\left[R_{\left(\alpha_{1}, 0, \cdots, 0\right)}\right]$ to $p_{1}\left[R^{\prime}\right]$.

Repeating such operations, we finally get $\alpha \in C^{2 g-2}$ such that $\alpha_{2 g-2}=0$ and that $\left[R_{\alpha}\right]=\left[R^{\prime}\right]$. Also, when $U$ is sufficiently small, $U$ is contained in $W_{\varepsilon}$, which implies the assertion. q.e.d.

Finally, next Lemma 3 completes the proof of Theorem 1.

LEMMA 3. The map $\Pi$ is a biholomorphic map of $E_{\varepsilon}$ onto its image.

Proof. Holomorphy of $\Pi$ follows from holomorphic dependence of $\mu(\alpha)$ on $\alpha$. Since $\Pi$ is injective by Lemma 1 , the assertion follows by a standard argument (cf. for instance [4, Lemma 6.13]). q. e.d.

Remark. We can further conclude that $R_{\alpha}=R_{\alpha^{\prime}}$ if and only if $\alpha_{\jmath}=\alpha_{j}{ }^{\prime}+c$ for every $j$ with a suitable $c$.

In particular, $\Pi\left(\left\{\alpha \in C^{2 g-2}|| \alpha \mid<\varepsilon / 2\right\}\right)$ is contained in $W_{\varepsilon}$.

\section{§3. An example.}

Even when the given differential $\varphi$ has a non-simple zero $p$, period-preserving variation at $p$ can be defined. Note that in case of a holomorphic abelian differential $\varphi$ with a non-simple zero, local parameterization induced by periodpreserving variation is hybrid. It indicates not only the complex structure of the resulting surface but also the ordering of zeros of the resulting differential.

Instead of going into details, we will give a simple but typical example.

Example. Let $S$ be a three-sheeted branched covering surface of $\boldsymbol{C}-\{0\}$ defined by

$$
\left\{(z, w) \mid z \in C-\{0\}, w^{3}=z^{2}(z-1)\right\}
$$

Add another copy $\boldsymbol{C}-\{0\}$ to one sheet of $S$ by sewing crosswise along $[-\infty,-1]$. Then we have a planar Riemann surface $R$ with 5 punctures. And the differential $(1 / z) d z$ induces a holomorphic differential $\varphi$ on $R$ with one simple zero $p_{0}$ over $z=-1$ and one double zero $p_{1}$ over $z=1$. (This represents 2 incoming, 3 outgoing strings. Cf. [3].)

In this case, $T(R)$ is complex 2 -dimensional, and since $R$ is planar, $W_{\varphi}$ is 
coincident with $T(R)$, and hence is also complex 2-dimensional, where $W_{\varphi}$ is defined as in $\S 2$.

Now one way to define local parameters for period-preserving variation is achived by keeping "one of two zeros" at $p_{1}$ fixed. This parametrization is really simple and we can show a similar theorem as Theorem 1. But to clarify complexity caused by non-simple zeros, we will keep $p_{0}$ fixed, and consider period-preserving variation only at $p_{1}$. Namely, first take a local parameter $z$ around $p_{1}$ such that $z\left(p_{1}\right)=0$ and that $\varphi$ can be written as $z^{2} d z$. Fix $\alpha=\left(\alpha_{1}, \alpha_{2}\right)$ with a sufficiently small $|\alpha|$, set $\alpha_{1}=r e^{i \theta}$, and consider two segments $L_{\alpha_{1}}$ and $L_{\alpha_{1}}^{\prime}=L_{\beta \alpha_{1}}$ on the $z$-plane, where we set $\beta=e^{2 \pi \imath / 3}$ and

$$
L_{\alpha}=\left\{0 \leqq|z| \leqq|\alpha|, \quad \arg \left(\frac{z}{\alpha}\right) \equiv 0(\bmod 2 \pi)\right\}
$$

for every $\alpha$. Also set $\alpha_{2}=\rho e^{\imath \phi}$, and assume that $\phi+2 k \pi \notin[\theta, \theta+2 \pi / 3)$ for every $k \in \boldsymbol{Z}$ if $\alpha_{1} \neq 0$. Consider two segments $L_{\alpha_{2}}$ and $L_{\alpha_{2}}^{\prime}$, where we set $L_{\alpha_{2}}^{\prime}=L_{\beta \alpha_{2}}$ if $\phi+2 \pi / 3+2 k \pi \notin(\theta, \theta+2 \pi / 3)$ for every $k \in \boldsymbol{Z}$, and $L_{\alpha_{2}}^{\prime}=L_{\bar{\beta} \alpha_{2}}$ otherwise.

Now, first cut the $z$-plane along $L_{\alpha_{1}}$ and $L_{\alpha_{1}}^{\prime}$, and paste all sides of cuts similarly as in $\S 1$. Second, cut along $L_{\alpha_{2}}$ and $L_{\alpha_{2}}^{\prime}$, and paste sides of cuts again similarly as before. (Here we need some carefulness for the pasting when $\theta-\phi \equiv 2 \pi / 3$ or $\equiv-2 \pi / 3$ modulo $2 \pi$.

The above surgery gives period-preserving variation at $p_{1}$ with respect to $\alpha=\left(\alpha_{1}, \alpha_{2}\right)$. We denote the resulting marked surface by $R_{\alpha}$.

Remark. Note that the map $\Pi: \alpha \rightarrow\left[R_{\alpha}\right]$ is never injective in this case. For instance, when $\phi+2 k \pi \notin(\theta-2 \pi / 3, \theta+2 \pi / 3)$ for every $k \in Z$, it is clear that $\left[R_{\left(\alpha_{1}, \alpha_{2}\right)}\right]=\left[R_{\left(\alpha_{2}, \alpha_{1}\right)}\right]$. Also, $\left[R_{\left(\alpha_{1}, \alpha_{2}\right)}\right]=\left[R_{\left(\alpha_{1}, \tilde{\alpha}_{2}\right)}\right]$ if $\tilde{\alpha}_{2}=\beta \alpha_{2}$ or $=\bar{\beta} \alpha_{2}$, except for the case that $\left|\alpha_{2}\right|>\left|\alpha_{1}\right|$ and $\left\{\alpha_{2}, \tilde{\alpha}_{2}\right\}=\left\{\lambda \beta \alpha_{1}, \lambda \bar{\beta} \alpha_{1}\right\}$ with some $\lambda>1$. (Noting these equivalent relations and Theorem 2 below, we can give a local parameter system at $[R]$.

On the other hand, we can show, similarly as in the proof of Lemma 2, the following.

THEOREM 2. Fix a sufficiently small $\varepsilon>0$, the set $\left\{R_{\alpha} \in T(R) \mid \alpha\right.$ is as above with $|\alpha|<\varepsilon\}$ covers a neighborhood of $[R]$ in $T(R)$.

\section{REFERENCES}

[1] R. D. M. Accola, Differentials and extremal length on Riemann surfaces, Proc. Nat. Acad. Sc1. U.S. A., 46 (1960), 540-543.

[2] F. GARDINER, Schiffer's interior variation and quasiconformal mappings, Duke Math. J. 42 (1975), 371-380. 
[3] S. Giddings And S. Wolpert, A triangulation of moduli space from light-cone string theory, Commun. Math. Phys., 109 (1987), 177-190.

[4] Y. Imayoshi And M. TANiguchi, An introduction to Teichmüller spaces, SpringerVerlag, 1992.

[5] M. TANigUchi, Certain kinds of convergence of holomorphic abelian differentials on the augmented Teichmüller spaces, J. Math. Kyoto Univ., 22 (1982), 293305.

[6] M. Taniguchi, Dirichlet finite harmonic differentials with integral periods on arbitrary Riemann surfaces, ibid., 23 (1983), 357-367.

Department of Mathematics

KYOTO UNIVERSITY 
